\title{
SINGULARITIES OF A CLASS OF MEROMORPHIC FUNCTIONS
}

\author{
NICHOLAS P. CALLAS AND W. J. THRON
}

Abstract. Estimates are obtained for the number of singular points, which are not poles, lying on the unit circle of the complex plane of a class of meromorphic functions which are represented by $C$-fractions.

I. Introduction. Representations of functions in terms of continued fractions. Given two sequences $\left\{a_{n}\right\}$ and $\left\{b_{n}\right\}$ of functions of a complex variable $z$, the continued fraction

$$
b_{0}(z)+\underset{n=1}{\infty}\left(\frac{a_{n}(z)}{b_{n}(z)}\right)
$$

is defined to be the sequence $\left\{A_{n}(z) \mid B_{n}(z)\right\}$, where the $n$th approximant

$$
\frac{A_{n}(z)}{B_{n}(z)}=b_{0}(z)+\frac{a_{1}(z)}{b_{1}(z)}+\frac{a_{2}(z)}{b_{2}(z)}+\cdots+\frac{a_{n}(z)}{b_{n}(z)} .
$$

If $A_{-1}=1, A_{0}=b_{0}, B_{-1}=0$, and $B_{0}=1$ and proper choices are made for arbitrary multiplicative constants, then the polynomials $A_{n}$ and $B_{n}$ satisfy the second order linear difference equations

$$
\begin{aligned}
& A_{n}(z)=b_{n}(z) A_{n-1}(z)+a_{n}(z) A_{n-2}(z), \\
& B_{n}(z)=b_{n}(z) B_{n-1}(z)+a_{n}(z) B_{n-2}(z) .
\end{aligned}
$$

Various representations of functions by classes of continued fractions have been investigated. Most important are those methods which provide for each formal power series expansion

$$
1+\sum_{n=1}^{\infty} c_{n} z^{n}
$$

exactly one continued fraction which corresponds to the series. A continued fraction (1) is said to correspond to a power series (3) if the power series expansion of $A_{n}(z) / B_{n}(z)$ agrees with (3) up to and including the term $c_{v(n)} z^{v(n)}$, where $v(n) \rightarrow \infty$ as $n \rightarrow \infty$.

Received by the editors April 9, 1971.

AMS 1969 subject classifications. Primary 3025, 4012; Secondary 3060.

$K e y$ words ard phrases. Continued fraction, $C$-fraction, $T$-fraction, $P$-fraction, meromorphic function.

(c) American Mathematical Society 1972 
The most recent are the $P$-fractions introduced by Magnus in [7] and further developed by him in [8], [9] and [10]. They are of interest in particular because of their close relation to Padé tables. A continued fraction (1) is a $P$-fraction if all $a_{n}(z)=1$ and the $b_{n}(z)$ are all polynomials in $z^{-1}$. The $T$-fractions were introduced by Thron [13] in 1948. These continued fractions are of the form $a_{n}(z)=z$ and $b_{n}(z)=1+d_{n} z$. They are of interest, among other reasons, because in them the elements $a_{n}(z)$ and $b_{n}(z)$ are linear functions of $z$. This enables one to establish fairly general convergence criteria for $T$-fractions. Recent work on $T$-fractions includes results of Waadeland [16], [17], [18] on convergence of $T$-fraction expansions of certain functions holomorphic in circular discs, as well as articles by Jones and Thron [5], Jefferson [4] and Hag [3]. Jones and Thron give, among other results, some theorems on the location of singular points of functions represented by $T$-fractions all of whose elements $d_{n}$ are positive.

In 1939, Leighton and Scott [6] introduced the $C$-fraction, defined by $b_{n}(z)=1$ and $a_{n}(z)=d_{n} z^{\alpha_{n}}$, where the $d_{n}$ are nonzero complex constants and the $\alpha_{n}$ are positive integers. Moduli of singular points of regular $C$-fractions were investigated by the present authors [1]. A central problem in the area of $C$-fractions is the conjecture of Leighton that meromorphic functions represented by them have the unit circle as a natural boundary, provided that $\alpha_{n} \rightarrow \infty$, and that the coefficients $d_{n}$ are suitably restricted, for example by

$$
\lim _{n \rightarrow \infty}\left(4\left|d_{n}\right|\right)^{1 / \alpha_{n}}=1,
$$

which would insure convergence of the $C$-fraction to a meromorphic function for $|z|<1$. This conjecture led to a series of investigations by Scott and Wall [11], Thron [14], [15], Singh and Thron [12] and Callas and Thron [2] which give successively better approximations to the conjecture of Leighton.

In the present paper we give substantial improvement over the previous best estimate [2] concerning the number of singular points lying on the unit circle of a class of meromorphic functions represented by $C$-fractions.

II. Singularities of meromorphic functions defined on the unit disc. Since we shall consider only $C$-fractions in the sequel, then in all cases $b_{n}(z)=1$.

If (4) holds, then it follows from the Worpitzky criterion $[19$, p. 42] that the $C$-fraction converges to a meromorphic function $f$ for all $z$ on the open unit disc.

Let $A_{n}^{(m) *}(z) / B_{n}^{(m) *}(z)$ be the $n$th approximant of the $C$-fraction (1), with $a_{n}(z)=\left|d_{m+n-1}\right| z^{x_{m+n-1}}$. Let $\sigma_{n}^{(m)}$ and $\tau_{n}^{(m)}$ be the degrees of the polynomials $A_{n}^{(m)}(z)$ and $B_{n}^{(m)}(z)$, respectively, where $A_{n}^{(m)}(z) / B_{n}^{(m)}(z)$ is the $n$th approximant of the $C$-fraction (1), with $a_{n}(z)=d_{m+n-1} z^{z_{m+n-1}}$. Let $\rho_{n}^{(m)}$ be 
the maximum of the degrees of $A_{n}^{(m) *}(z)$ and $B_{n}^{(m) *}(z)$. Then $\sigma_{n}^{(m)} \leqq \rho_{n}^{(m)}$ and $\tau_{n}^{(m)} \leqq \rho_{n}^{(m)}$.

Further, let

$$
h_{n}=h_{n}^{(1)}, \quad h_{n}^{(m)}=\sum_{v=m}^{n+1} \alpha_{v}
$$

(6) $k=k^{(1)}$

$$
k^{(m)}=\liminf _{v \rightarrow \infty} k_{v}^{(m)}, \quad k_{v}^{(m)}=\frac{\rho_{n_{v}}^{(m)}}{i_{n_{v}}^{(m)}-\rho_{n_{v}}^{(m)}} ;
$$

and

$$
N=N^{(1)}, \quad N^{(m)}=\limsup _{v \rightarrow \infty} N_{v}^{(m)}, \quad N_{v}^{(m)}=\frac{n_{v}-m+2}{h_{n_{v}}^{(m)}-\rho_{n_{v}}^{(m)}} .
$$

Using these definitions, the main result of this paper is stated as follows:

THEOREM 1. For the C-fraction (1), let $a_{n}(z)=d_{n} z^{\alpha_{n}}$, where $d_{n} \neq 0$ are all complex numbers and $\alpha_{n}$ are all positive integers. Suppose that there exists a sequence of positive integers $\left\{n_{v}\right\}$ such that $N=0$. Let condition (4) be satisfied and suppose $k>0$, defined by (6), is finite. Then the meromorphic function $f$ to which (1) converges on the unit disc has at least $[(1+k) / k]+1$ singular points, which are not poles, on the unit circle. Further, $f$ cannot be meromorphic on any arc of the unit circle of angular measure greater than $2 \pi k /(1+k)$ radians.

REMARKS. Several sufficient conditions for the existence of sequences $\left\{n_{v}\right\}$ which insure that $N=0$ are given in [2].

In comparing the above result with the previous best result, consider the parameter $k$ such that $k<10^{-2 s}$, where $s$ is the largest such positive integer. An estimate of a lower bound for the number of singular points given in [2] is then $S_{1}=10^{s}$. However the result produced by Theorem 1 is the estimate $S_{2}=10^{28}$. Thus the present result gives the improvement $S_{2}=S_{1}^{2}$.

ProOF OF THEOREM 1. We start by stating two lemmas which are proved in [2] and [12], respectively:

LEMMA 2. Let the power series $P(w)=\sum_{v=0}^{\infty} p_{v} w^{v}$ have radius of convergence greater than $1+\varepsilon$, where $\varepsilon>0$. Define polynomials

Let

$$
B(z)=\sum_{v=0}^{N} b_{v} z^{v} \text { and } B^{*}(z)=\sum_{v=0}^{N}\left|b_{v}\right| z^{v}
$$

$$
g(w)=P(w) B(\phi(w))=\sum_{n=0}^{\infty} c_{n} w^{n}
$$


where $z=\phi(w)$ is holomorphic in a domain containing the closed disc $|w| \leqq$ $1+\varepsilon$, and such that

Then

$$
\max _{0 \leqq \theta<2 \pi}\left|\phi\left((1+\varepsilon) e^{i \theta}\right)\right|=\phi(1+\varepsilon)>0 .
$$

$$
\left|c_{n}\right| \leqq \frac{M B^{*}(\phi(1+\varepsilon))}{(1+\varepsilon)^{n}}, \text { where } M=\max _{|w|=1+\varepsilon}|P(w)| .
$$

LEMMA 3. If $\left\{n_{v}\right\}$ is a sequence such that $N=0$, then $N^{(m)}=0$ and $k^{(m)}=k$ for all $m$.

Before presenting four more lemmas to be used in the proof of Theorem 1 , we shall define the auxiliary function $\varphi$ as follows: Let $R>1$ and $\varepsilon>0$. Then $\varphi(w, \rho)=w\{1+D(w) \rho\}$, where $\rho$ is real, $|w| \leqq 1+\varepsilon<R$, and

with

$$
D\left(r e^{i \theta}\right)=u_{1}(r, \theta)+i u_{2}(r, \theta),
$$

$$
u_{v}(r, \theta)=\frac{1}{2 \pi} \int_{0}^{2 \pi} U(\zeta) K_{v}(r, \theta ; R, \zeta) d \zeta
$$

for $\nu=1,2$, where

and

$$
\begin{aligned}
& K_{1}(r, \theta ; R, \zeta)=\frac{R^{2}-r^{2}}{R^{2}+r^{2}-2 r R \cos (\theta-\zeta)}, \\
& K_{2}(r, \theta ; R, \zeta)=\frac{2 r R \sin (\theta-\zeta)}{R^{2}+r^{2}-2 r R \cos (\theta-\zeta)},
\end{aligned}
$$

$$
\begin{aligned}
U(\zeta) & =U(0)>0, & & 0 \leqq \zeta \leqq \zeta_{1}, \\
& =U(0)\left(\zeta-\zeta_{0}\right) /\left(\zeta_{1}-\zeta_{0}\right), & & \zeta_{1} \leqq \zeta \leqq \zeta_{0}, \\
& =-\left(\zeta-\zeta_{0}\right) /\left(\zeta_{2}-\zeta_{0}\right), & & \zeta_{0} \leqq \zeta \leqq \zeta_{2}, \\
& =-1, & & \zeta_{2} \leqq \zeta \leqq \pi,
\end{aligned}
$$

where $U(\zeta)=U(2 \pi-\zeta)$ and $U(\zeta)=U(\zeta+2 \pi)$ for all $\zeta$. Further, we require that the values $U(0)$ and $\zeta_{0}$ satisfy the relations

(8) $\zeta_{0} U(0)=\pi-\zeta_{0}$ and $k U(0)<1-\delta$, where $0<\delta<U(0)$.

It is easy to see that $D(\bar{w})=(D(w))^{-}$. Further, $\varphi$ is holomorphic in $w$ on the disc $|w| \leqq 1+\varepsilon<R$ for any $\rho$. Note that $\varphi(w, 0)=w$. For notational convenience, in the sequel, we shall write $u_{1}=u$ and $u_{2}=v$.

Lemma 4. Let $\delta>0$. Then, for sufficiently small $R>1$,

$$
|u(1+\varepsilon, \theta)-U(\theta)|<\delta
$$

for all $\theta$ such that $0 \leqq \theta \leqq 2 \pi$ and for any $\varepsilon$ such that $1 \leqq 1+\varepsilon<R$. 
Proof. This lemma follows easily from the continuity of $U$ on the compact set $0 \leqq \theta \leqq 2 \pi$, which implies that the Poisson integral $u(r, \theta)$ satisfies $\lim _{r \rightarrow R} u(r, \theta)=U(\theta)$ uniformly with respect to $0 \leqq \theta \leqq 2 \pi$.

As consequences of this lemma, it is easy to see that $D(1)=U(0)$ and $D(-1)=-1$, where $D(1)=u(1,0)$ and $D(-1)=u(1, \pi)$.

LEMMA 5. For all sufficiently small $\rho>0$,

$$
\max _{0 \leqq \theta<2 \pi}\left|q\left((1+\varepsilon) e^{i \theta}, \rho\right)\right|=\varphi(1+\varepsilon, \rho)
$$

for all $\varepsilon$ such that $1 \leqq 1+\varepsilon \leqq R_{1}<R$.

Proof. For the moment let us assume, for all $\theta$ such that $0 \leqq \theta<2 \pi$, that

$$
\operatorname{Re}\left\{D\left((1+\varepsilon) e^{i \theta}\right)\right\} \leqq D(1+\varepsilon),
$$

with equality only at $\theta=0$. Then, for all $\varepsilon$ such that $1+\varepsilon \leqq R_{1}<R$ and all sufficiently small $\rho$, we can easily prove that

$$
2 \operatorname{Re}\left\{D\left((1+\varepsilon) e^{i \theta}\right)\right\}+\left|D\left((1+\varepsilon) e^{i \theta}\right)\right|^{2} \rho \leqq 2 D(1+\varepsilon)+D(1+\varepsilon)^{2} \rho
$$

for all $\theta$ on $\sigma \leqq \theta \leqq 2 \pi-\sigma$, where $\sigma>0$. From the identity

$$
\begin{aligned}
& \left|\varphi\left((1+\varepsilon) e^{i \theta}, \rho\right)\right|^{2} \\
& \quad=(1+\varepsilon)^{2}\left\{1+2 \operatorname{Re}\left\{D\left((1+\varepsilon) e^{i \theta}\right)\right\} \rho+\left|D\left((1+\varepsilon) e^{i \theta}\right)\right|^{2} \rho^{2}\right\}
\end{aligned}
$$

it is clear that if we show that (10) holds on the whole interval $0 \leqq \theta<2 \pi$, then we will have proved the lemma, provided, of course, that we prove (9).

To show that $(10)$ holds on the interval $0 \leqq \theta<2 \pi$, we consider this inequality in terms of $u$ and $v$. Since we have assumed for the moment that (9) holds, with equality only at $\theta=0$, then

(11) $2 \geqq \rho\left\{\frac{v(1+\varepsilon, \theta)^{2}}{u(1+\varepsilon, 0)-u(1+\varepsilon, \theta)}-u(1+\varepsilon, \theta)-u(1+\varepsilon, 0)\right\}$

for $\theta \neq 0$. By Taylor expansion of $u(1+\varepsilon, \theta)$ and $v(1+\varepsilon, \theta)$ about $\theta=0$, the first term in the brackets of (11) becomes

$$
\frac{2 v_{\theta}(1+\varepsilon, 0)^{2}}{-u_{\theta \theta}(1+\varepsilon, 0)}+O(\theta),
$$

provided $u_{\theta \theta}(1+\varepsilon, 0) \neq 0$. If $u_{\theta \theta}(1+\varepsilon, 0) \neq 0$, ther $\rho$ can be chosen so small that the inequality (11) holds, and hence (10) holds for all $\theta$ such that $0 \leqq$ $\theta<2 \pi$. The inequality $u_{\theta \theta}(1+\varepsilon, 0 ; \neq 0$ can be established by first proving the Riemann-Stieltjes integral representation

$$
u_{\theta \theta}(1+\varepsilon, \theta)=\frac{1}{2 \pi} \int_{0}^{2 \pi} K_{1}(1+\varepsilon, \theta ; R, \zeta) d U^{\prime}(\zeta)
$$


Then, for $\theta=0$, it follows easily that $K\left(\zeta_{1}\right)>K\left(\zeta_{0}\right)>K\left(\zeta_{2}\right)$, where $K(\zeta)=$ $K_{1}(1+\varepsilon, 0 ; R, \zeta)$, implies that $u_{\theta \theta}(1+\varepsilon, 0)<0$.

To complete the proof of the lemma it suffices to show that (9) holds with equality only at $\theta=0$. This can be done by proving that $u(1+\varepsilon, \theta)$ is strictly decreasing on $0<\theta<\pi$ and strictly increasing on $\pi<\theta<2 \pi$, and that $u_{\theta}(1+\varepsilon, 0)=u_{\theta}(1+\varepsilon, \pi)=0$. This completes the proof of the lemma.

LeMMA 6. Let $1 \leqq 1+\varepsilon<R$ and $u^{*} \in(\delta-1,0)$, where $0<\delta<\min \{1, U(0)\}$ and $R=R(\delta)$ is as in Lemma 4. Then there exists a unique $\theta^{*} \in\left(\zeta_{1}, \zeta_{2}\right)$ such that $u\left(1+\varepsilon, \theta^{*}\right)=u^{*}$.

Proof. Lemma 4 implies that $u\left(1+\varepsilon, \zeta_{2}\right)<\delta-1$ and $u\left(1+\varepsilon, \zeta_{1}\right)>$ $U(0)-\delta>0$. Further, $u^{*} \in(\delta-1,0)$ implies that there exists a $\theta^{*}$ such that $u\left(1+\varepsilon, \theta^{*}\right)=u^{*}$. Uniqueness follows from the fact stated in Lemma 5 that $u(1+\varepsilon, \theta)$ is strictly decreasing on the open interval $(0, \pi)$. Moreover, this monotonicity property of $u$ implies that $\zeta_{2}>\theta^{*}>\zeta_{1}$. This concludes the proof of the lemma.

LEMMA 7. Let $f$ be the meromorphic function to which the $C$-fraction (1) converges. Define $F$ by the composition $F(w, \rho)=f(\varphi(w, \rho))$, where $\varphi(w, \rho)$, as defined before, is holomorphic in $w$ with $\rho$ sufficiently small and $R>1$ such that $R-1$ is sufficiently small. Let $\Delta=\varphi(1+\varepsilon, \rho) /(1+\varepsilon)$. Assume that $F(w, \rho)$ is meromorphic for $|w|<1+\varepsilon^{*}$ and let $0<\varepsilon<\varepsilon^{*}$. Then $1+\varepsilon \leqq \Delta^{k}$, where $k$ is defined by (6).

Proof. The meromorphic function $f$ may be written as $f(z)=p(z) / q(z)$, where $p$ and $q$ are holomorphic wherever $f$ is meromorphic and $p(0)=$ $q(0)=1$. It is well known [12] that

$$
p(z) B_{n}(z)-q(z) A_{n}(z)=(-1)^{n}\left(\prod_{v=1}^{n+1} d_{v}\right) z^{h_{n}}+\cdots,
$$

where the omitted terms on the right are of degree higher than $h_{n}$ in $z$. Let $\pi_{n}=\prod_{v=1}^{n+1} d_{v}$, where the product is taken only over those factors for which $\left|d_{v}\right|>1$. Let $A_{n}^{\prime}(z)=A_{n}(z) / \pi_{n}$ and $B_{n}^{\prime}(z)=B_{n}(z) / \pi_{n}$. Then the coefficients of the polynomials $A_{n}^{\prime}(z)$ and $B_{n}^{\prime}(z)$ satisfy [12]

$$
\left|a_{v}^{(n)}\right| \leqq 2^{n} \text { and }\left|b_{v}^{(n)}\right| \leqq 2^{n}
$$

for $v \leqq \sigma_{n}$ and $v \leqq \tau_{n}$, respectively. Define the compositions $P(w, \rho)=$ $p(\varphi(w, \rho))$ and $Q(w, \rho)=q(\varphi(w, \rho))$. Then $P(0, \rho)=Q(0, \rho)=1$, and $P$ and $Q$ are holomorphic in $w$ wherever $F$ is meromorphic. The identity (12) becomes, upon letting $z=\varphi(w, \rho)$,

$$
\begin{aligned}
P(w, \rho) B_{n}^{\prime}(\varphi(w, \rho))-Q & (w, \rho) A_{n}^{\prime}(\varphi(w, \rho)) \\
& =(-1)^{n}\left(\prod_{v=1 ;\left|d_{v}\right| \leqq 1}^{n+1} d_{v}\right) w^{h_{n}}+\cdots,
\end{aligned}
$$


where the omitted terms are of higher degree than $h_{n}$ in $w$. Let $P$ and $Q$ have power series expansions

$$
P(w, \rho)=1+\sum_{v=1}^{\infty} p_{v} w^{v} \text { and } Q(w, \rho)=1+\sum_{v=1}^{\infty} q_{v} w^{\nu} .
$$

Then both these series have radii of convergence greater than $1+\varepsilon$. By Lemmas 2 and 5, if

and

$$
P(w, \rho) B_{n}^{\prime}(\varphi(w, \rho))=\sum_{v=0}^{\infty} c_{v}^{(n)} w^{v}
$$

then

$$
Q(w ; \rho) \cdot A_{n}^{\prime}(\varphi(w, \rho))=\sum_{v=0}^{\infty} e_{v}^{(n)} w^{v}
$$

$$
\left|c_{v}^{(n)}\right| \leqq \frac{M_{1} B_{n}^{\prime \prime}(\varphi(1+\varepsilon, \rho))}{(1+\varepsilon)^{v}} \text { and }\left|e_{v}^{(n)}\right| \leqq \frac{M_{2} A_{n}^{\prime \prime}(q(1+\varepsilon, \rho))}{(1+\varepsilon)^{v}}
$$

for $y=0,1,2, \cdots$, where

$$
M_{1}=\max _{|w|=1+\varepsilon}|P(w, \rho)| \text { and } M_{2}=\max _{|w|=1+\varepsilon}|Q(w, \rho)|
$$

and $A_{n}^{\prime \prime}(z)$ and $B_{n}^{\prime \prime}(z)$ are the polynomials obtained from $A_{n}^{\prime}(z)$ and $B_{n}^{\prime}(z)$, respectively, where each coefficient is replaced by its modulus. Let $M=$ $\max \left\{M_{1}, M_{2}\right\}$. Then it follows from the identity (14), by using the estimates (13) and (15), that

$$
\prod_{v=1 ;\left|\partial_{v}\right| \leqq 1}^{n+1}\left|d_{v}\right| \leqq \frac{2^{n+1} M}{(1+\varepsilon)^{h_{n}}} \frac{\{(1+\varepsilon) \Delta\}^{\rho_{n+1}}}{(1+\varepsilon) \Delta-1} .
$$

In a similar manner for the $C$-fraction (1), with $a_{n}(z)=d_{m+n-1} z^{\alpha_{m+n-1}}$, where the $d_{v}$ satisfy (4), then, for fixed arbitrary $\eta$ such that $0<\eta<1$ and sufficiently large $m$ depending on $\eta$,

$$
\frac{(1-\eta)^{h_{n}^{(m)}}}{4^{n-m+2}} \leqq \prod_{v=m ;\left|d_{v}\right| \leqq 1}^{n+1}\left|d_{v}\right| \leqq \frac{2^{n-m+2} M^{\prime}}{(1+\varepsilon)^{h_{n}^{(m)}}} \frac{\{(1+\varepsilon) \Delta\}_{n}^{\rho_{n}^{(m)}+1}}{(1+\varepsilon) \Delta-1} .
$$

Let $\left\{n_{v}\right\}$ be the sequence in Lemma 3 , then $k=k^{(m)}$ and $N^{(m)}=0$ for all $m$. Taking the $\left(h_{n_{v}}^{(m)}-\rho_{n_{v}}^{(m)}\right)$ th roots of the extreme members of the inequalities (16) and letting $\nu \rightarrow \infty$, we get $(1-\eta)^{1+k} \leqq \Delta^{k} /(1+\varepsilon)$. Since $\eta>0$ is ar bitrary, the lemma is proved.

The proof of Theorem 1 now shall be completed in two steps. First, an arc $\Gamma$ on the unit circle will be found which is not free of singular points of the function $f$. Second, we shall calculate the angular measure of the arc $\Gamma$ to be $2 \pi k /(k+1)$ radians. Then the theorem follows easily by a rotation 
argument which proves that no arc of that angular measure is free of singular points.

Let $\varepsilon>0$ be the largest value for which $F(w, \rho)$ is meromorphic on the open disc $|w|<1+\varepsilon$, then $1+\varepsilon \leqq\{1+D(1+\varepsilon) \rho\}^{k}$. Hence $k^{-1} \varepsilon+T(\varepsilon) \leqq$ $D(1+\varepsilon) \rho$, where $T(0)=0$ and $T(\varepsilon) / \varepsilon \rightarrow 0$ as $\varepsilon \rightarrow 0$. Define $\rho=X(\varepsilon)=$ $\left(k^{-1} \varepsilon+T(\varepsilon)\right) / D(1+\varepsilon)$. Then $X^{\prime}(0)=1 / k D(1)>0$. Let $\rho^{*}=X^{*}(\varepsilon)$ such that $X^{*}$ is a twice continuously differentiable function, $X^{\prime}(0)=X^{*^{\prime \prime}}(0)$, and $X(\varepsilon)>X^{*}(\varepsilon)$ in a neighborhood of $\varepsilon=0$. Then, for all $\varepsilon>0$ in this neighborhood,

$$
1+\varepsilon=\{1+D(1+\varepsilon) \rho\}^{k}>\left\{1+D(1+\varepsilon) \rho^{*}\right\}^{k} .
$$

Hence, for any sufficiently small $\varepsilon>0, F\left(w, \rho^{*}\right)$ cannot be meromorphic on the open disc $|w|<1+\varepsilon$. Since $q\left(w, \rho^{*}\right)$ is holomorphic on $|w|<1+\varepsilon$, then the singular points of $F\left(w, \rho^{*}\right)$ in the region $|w| \leqq 1+\varepsilon$ are caused by the singular points of the function $f$ in the image region $\varphi\left(G, \rho^{*}\right)$, where $G=\{w:|w| \leqq 1+\varepsilon\}$. Since $f$ has no singular points, other than poles, on the unit disc $V$, then its singularities lie in the crescent-shaped region $\varphi\left(G, \rho^{*}\right)-$ $V$, assuming that there is just one intersection point of the image of the boundary of $G$ with the unit circle in the upper half plane. As $\varepsilon \rightarrow 0$, this crescent-shaped region contracts to an arc $\Gamma$ on the unit circle, which is symmetric about the point $z=1$. Therefore, there is at least one singular point of $f$ on the arc $\Gamma$.

In calculating the angular measure of this arc $\Gamma$, for sufficiently small $\varepsilon>0$, let $\theta(\varepsilon)$ satisfy the equation

$$
\left|\varphi\left((1+\varepsilon) e^{i \theta(\varepsilon)}, X^{*}(\varepsilon)\right)\right|=1,
$$

where $\theta(\varepsilon)>0$ is taken as the smallest such solution. That such a solution exists follows from the intermediate value theorem and the inequalities

$$
-1<\varphi\left(-(1+\varepsilon), X^{*}(\varepsilon)\right)<1<\varphi\left(1+\varepsilon, X^{*}(\varepsilon)\right) .
$$

Conditions which imply that (18) hold are the following: The equality $D(-1)=-1$ implies that the left-most inequality of (18) holds. Further, since we shall require that $1-k U(0)>0$ be arbitrarily small in the sequel, then the middle inequality of (18) will be satisfied also. The right-most inequality of (18) holds without conditions beyond the requirement that $k>0$.

By implicit differentiation of the squared members of the defining relation (17) of $\theta(\varepsilon)$ with respect to $\varepsilon$, we get, upon letting $\varepsilon \rightarrow 0$, the necessary condition $u\left(1, \theta_{0}\right)=-k D(1)$. Let $u^{*}=-k D(1)<0$. Since $D(1)=U(0)$, then the condition $-k U(0)>\delta-1$ (see (8)) implies that $u^{*}>\delta-1$. Hence $\theta_{0}$ exists, and, by Lemma $6, \theta_{0}$ is unique and satisfies $\zeta_{1}<\theta_{0}<\zeta_{2}$. Therefore, by a continuity argument, for sufficiently small $\varepsilon>0$, (17) has a unique solution $\theta(\varepsilon)$. 
Let $\lambda=1-k U(0)$. Then $0<\delta<\lambda$, where $\delta$ is the modulus of uniform convergence arbitrarily specified in Lemma 4 . Solving for $\zeta_{0}$ in the equation of (8) gives $\zeta_{0}=\pi /(U(0)+1)$. Then, using the definition of $\lambda_{\text {. above, }}$ $\check{\zeta}_{0}=k \pi /(1+k-i)$. Since $\delta>0$ is arbitrary and $\lambda$ may be chosen as small as we please such that $\delta<\lambda$ and $\zeta_{2}-\zeta_{1}$ may be taken as small as we please, then $\theta_{0} \approx \pi k /(k+1)$ with any degree of accuracy desired.

Hence the angular measure of the arc $\Gamma$ is $2 \pi k /(k+1)$, since by letting $e^{i \gamma(\theta(\varepsilon))}$ be the image of $w=(1+\varepsilon) e^{i \theta(\varepsilon)}$ under the transformation $\varphi\left(w^{\prime}, X^{*}(\varepsilon)\right)$, then the fact that $\lim _{\varepsilon \rightarrow 0} \psi\left(w, X^{*}(\varepsilon)\right)=w$ uniformly on $|w| \leqq R_{1}$ implies that $\lim _{\varepsilon \rightarrow 0} \gamma(\theta(\varepsilon))=\theta_{0}(\bmod 2 \pi)$. This completes the proof of Theorem 1 .

\section{REFERENCES}

1. N. P. Callas and W. J. Thron, Singularities of meromorphic funcrions represented by regular C-fractions, Norske Vid. Selsk. Skr. (Trondheim) 1967, no. 6. MR 36 \#6595.

2. - Singular points of certain functions represented by C-fractions, J. Indian Math. Soc. 32 (1968), suppl. 1, 325-353.

3. Kari Hag, $A$ thenrem on $T$-fractions corresponding to a rational function, Proc. Amer. Math. Soc. 25 (1970), 247-253. MR 41 \#3723.

4. Thomas H. Jefferson, Truncation error estimates for T-fractions, SIAM J. Numer. Anal. 6 (1969), 359-364. MR 41 \#4775.

5. William B. Jones and W. J. Thron, Further properties of T-fractions, Math. Ann. 166 (1966), 106-118. MR 34 \#319.

6. Walter Leighton and W. T. Scott, A generai continued fraction expansion, Bull. Amer. Math. Soc. 45 (1939), 596-605. MR 1, 7.

7. Arne Magnus, Certain continued fractions associated with the Padé table, Math. $\mathrm{Z}$. 78 (1962), 361-374. MR 27 \#272.

8. - Expansion of power series into P-fractions, Math. Z. 80 (1962), 209-216. MR 27 \#273.

9. - On P-expansions of power series, Norske Vid. Selsk. Skr. (Trondheim) 1964, no. 3, 14 pp. MR 32 \#1330.

10. - The connection between P-fractions and associated fractions, Proc. Amer. Math. Soc. 25 (1970), 676-679. MR 41 \#4050.

11. W. T. Scott and H. S. Wall, Continued fraction expansions for arbitrary power series, Ann. of Math. (2) 41 (1940), 328-349. MR 1, 296.

12. V. Singh and $\mathrm{W}$. J. Thron, On the number of singular points, located on the unit circle, of certain functions represented by C-fractions, Pacific J. Math. 6 (1956), 135-143. MR 18, 274.

13. W. J. Thron, Some properties of the continued fraction $\left(1+d_{0} z\right)+K\left(z /\left(1+d_{n} z\right)\right)$, Bull. Amer. Math. Soc 54 (1948), 206-218. MR 9, 508.

14. - Singular points of functions defined by $C$-fractions, Proc. Nat. Acad. Sci. U.S.A. 36 (1950), 51-54, MR 11, 429.

15. - A class of meromorphic functions having the unit circle as a natural boundary, Duke Math. J. 20 (1953), 195-198. MR 15, 113.

16. Haakon Waadeland, A convergence property of certain T-fraction expansions, Norske Vid. Selsk, Skr. (Trondheim) 1966, no. 9, 22 pp. MR 37 \#1568. 
17. Haakon Waadeland, On T-fractions of certain functions with a first order pole at the point of infinity, Norske Vid. Selsk. Forh. (Trondheim) 40 (1967), 1-6. MR 38 \#2289.

18. - On T-fractions of functions holomorphic and bounded in a circular disc, Norske Vid. Selsk. Skr. (Trondheim) 1964, no. 8, 19 pp. MR 31 \#1364.

19. H. S. Wall, Analytic theory of continued fractions, Van Nostrand, Princeton, N.J., 1948. MR 10, 32.

Air Force Office of Scientific Research, Arlington, Virginia 22209

Department of Mathematics, University of Colorado, Boulder, Colorado 80302 